\title{
Matrix Pencil Method: Angle of Arrival and Channel Estimation for a Massive MIMO system
}

\author{
Laura Monteyne*, Andrea P. Guevara*, Gilles Callebaut*, Sara Gunnarsson* ${ }^{*}$, \\ Liesbet Van der Perre* and Sofie Pollin* \\ *Department of Electrical Engineering, KU Leuven, Belgium \\ †Department of Electrical and Information Technology, Lund University, Sweden \\ E-mail: \{laura.monteyne, gilles.callebaut, liesbet.vanderperre, sofie.pollin\} @ kuleuven.be, \\ andrea.guevara@esat.kuleuven.be, sara.gunnarsson@eit.lth.se
}

\begin{abstract}
Channel estimation is essential in massive MIMO systems. Pilot Contamination (PC) however, causes a major bottleneck in the acquisition of this information. The exploitation of the Angle of Arrival (AoA) provides multiple techniques for channel estimation under PC. However, many AoA estimation techniques require information on the signal statistics which is not available in dynamic scenarios. In this paper we propose and analyse the Matrix Pencil Method (MPM) to decorrelate contaminated channels based on their estimated AoA. We evaluate this method both through simulations and experiments in a real-life testbed. Our assessment focuses on a system with a Uniform Linear Array (ULA). The performance of the MPM is validated through simulations ${ }^{1}$ with varying number of antennas, SNR and AoA difference. The results show that our approach effectively decorrelates the channels starting from 20 antennas and an SNR of $15 \mathrm{~dB}$, which outperforms the theoretical expectation. This allows us to enhance the channel estimation quality under PC to the level of no PC. Real-life measurements confirm the simulated results. Our MPM implementation can achieve a target AoA estimation accuracy both with and without PC. We anticipate that the method can be extended for a Uniform Rectangular Array (URA).
\end{abstract}

Index Terms-Massive MIMO, Matrix Pencil Method, pilot contamination, channel estimation, experimental validation

\section{INTRODUCTION}

Massive MIMO is a core technology to achieve the demanding requirements of $5 \mathrm{G}$ regarding spectral efficiency as well as energy efficiency. Massive MIMO enables multiple single-antenna User Equipments (UEs) to communicate with a Base Station (BS) using the same bandwidth and time [1]. To ensure reliable wireless communication, channel estimation is crucial. During channel estimation, each UE sends a known orthogonal sequence, a so-called pilot, to the BS. We focus on massive MIMO systems using Time-Division Duplexing (TDD) [2]. Channels in TDD systems are reciprocal, therefore, the channel estimation performed during uplink (UL) can be applied in downlink (DL) as well [3]. The number of possible orthogonal sequences is limited due to the finite coherence time and bandwidth. The reuse of pilot sequences, and thus $\mathrm{PC}$, is inevitable when there are more users than orthogonal pilots. Consequently, the channels of UEs sharing the same pi-

\footnotetext{
${ }^{1}$ We would like to thank NVIDIA for providing the GPU that was used to greatly accelerate our simulations.
}

lots are 'contaminated' during the channel estimation process, yielding channel estimation errors [2].

In [4] and [5] it is shown that the impact of PC could be reduced with a coordinated pilot allocation based on the AoA of each UE. For the generic problem of AoA estimation, multiple techniques have been proposed including the Matrix Pencil Method (MPM). The original contribution of our paper is to implement and analyse the MPM method for AoAbased pilot decontamination in massive MIMO. The MPM is a linearised prediction method that estimates the parameters of a sum of complex exponentials [6]. With the estimated poles, the AoA of the signal is estimated. Thereafter, the estimated AoA can be used to model and thus estimate the channel of a 'contaminated' signal. The MPM is expected to handle SNR values of 20-25 dB adequately [7]. For a lower SNR $(<12 \mathrm{~dB})$, the bandpass MPM can be applied [8], where the signal is pre-filtered. Alternative estimation methods for the AoA are MUSIC and ESPRIT. In this work we focus on the MPM as the preferred method in a non-static scenario because it requires only one snapshot, in contrast to MUSIC and ESPRIT [9].

This paper considers a TDD massive MIMO system with a single BS, equipped with a Uniform Linear Array (ULA) with $M$ antennas. We envision situations with a high user load, leading to situations where two users communicate simultaneously with the same BS under PC condition. However, we assume that the user load is limited such that there are never three UEs with the same pilot sequence. The main contribution of this paper to existing research is twofold. Firstly, we design an MPM-based approach for channel estimation in Massive MIMO. In particular, we consider scenarios with heavy user load which may result in PC between two of these users. Secondly, we assess the performance of the MPM based on Matlab simulations and experimentally validate these through real-life indoor measurements.

Section II elaborates on the theoretical study of the MPM as well as the simulation framework that evaluates the performance of the MPM. The results of these simulations are presented and discussed in section III. Furthermore, section IV introduces the measurement testbed with its possibilities and restrictions regarding this study. Results of these measurements are included as well. Finally, the main conclusions are summarised in section $\mathrm{V}$, along with future work. 


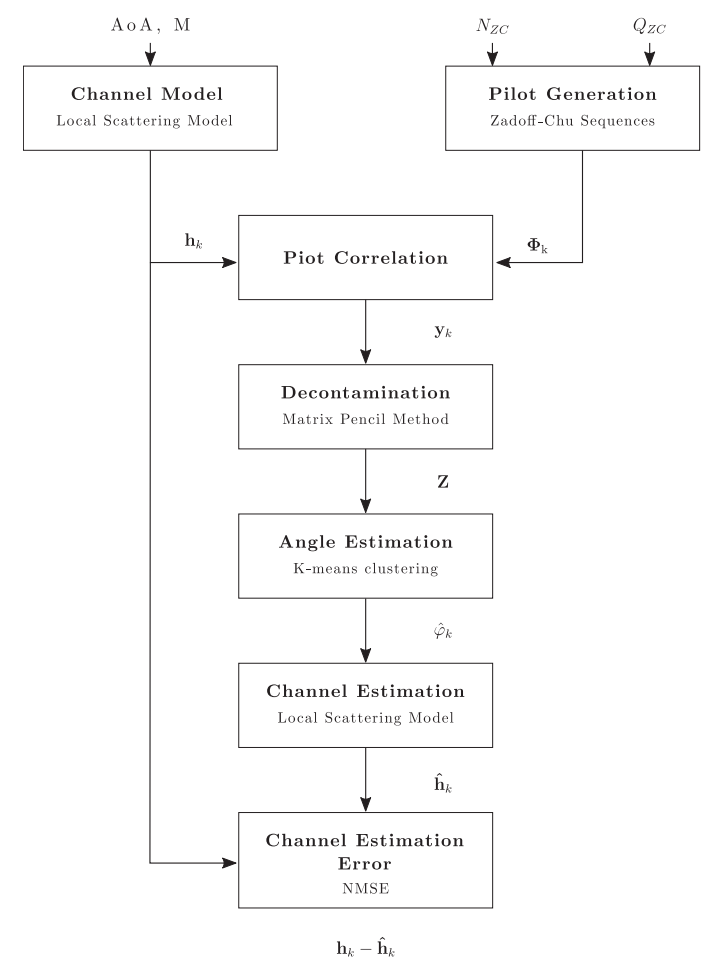

Fig. 1. Overview of the system analysis approach.

\section{SYSTEM DESIGN AND ANALYSIS}

An overview of the system design and analysis approach is given in Fig. 1. It contains the main functions with their input and output parameters, as further discussed in this section. Section II-A introduces the local scattering one-ring model and clarifies why it is suited for this analysis. Next, pilot training with Zadoff-Chu sequences is elaborated on in section II-B. We propose the MPM-based approach in section II-C as a method to decontaminate signals that suffer from PC by estimating the poles of each signal. The implementation of the MPM and its parameters are presented in section II-D. Finally, the resulting channel estimation solution and its assessment are treated in section II-E.

\section{A. Channel model}

The local scattering one-ring channel model [2] is selected because of its simplicity and direct link to the AoA $\varphi$. The steering vector is defined in [10] as

$$
\mathbf{a}\left(\theta_{n}\right)=\left[\begin{array}{llll}
1 & e^{-j 2 \pi d_{H} \theta_{n}} & \ldots & e^{-j 2 \pi d_{H}(M-1) \theta_{n}}
\end{array}\right]^{T}
$$

with

$$
\theta_{n}=\sin \left(\varphi+\delta_{n}\right)
$$

and where $[.]^{T}$ denotes the transpose operator. In (2), $\delta_{n}$ is the angular spread from a uniform distribution $U[-\sqrt{3} \sigma, \sqrt{3} \sigma]$ with standard deviation $\sigma$. This model assumes that all the scatterers are located on a circle around the UE. A typical value for the standard deviation is $10^{\circ}$ for an urban environment and a BS located on a rooftop, according to [11].

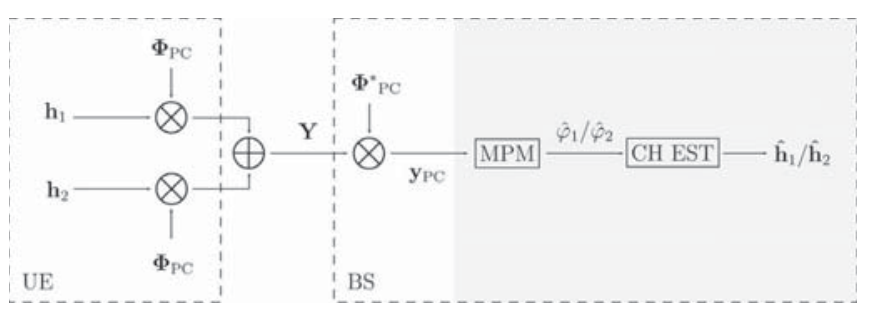

Fig. 2. Overview of pilot correlation, MPM and channel estimation. The gray shaded box indicates the contribution of this paper.

The subscript $n$ in 2 stands for the $n_{t h}$ path of in total $N$ paths. Because of the sine operation, the algorithm is suited to estimate values of $\varphi$ in the interval $\left[-90^{\circ}, 90^{\circ}\right]$. Another input parameter of the channel model is $d_{H}$, the antenna spacing in the ULA, which is the distance between two consecutive antennas in terms of number of wavelengths. In line with [2], this parameter is fixed to $\frac{1}{2} \lambda$.

To combine $N$ different paths, the matrix $\mathbf{A}_{R}$ at the receiver is constructed with dimension $M \times N$ as

$$
\mathbf{A}_{R}=\left[\begin{array}{llll}
\mathbf{a}\left(\theta_{1}\right) & \mathbf{a}\left(\theta_{2}\right) & \ldots & \mathbf{a}\left(\theta_{n}\right)
\end{array}\right] .
$$

With $\mathbf{H}_{p}$, a diagonal matrix containing the gains of the different paths and $\mathbf{1}_{N}$ a vector containing $N$ ones, the discrete physical channel $\mathbf{h}_{k}$ for each user $k$ is calculated as

$$
\mathbf{h}_{k}=\mathbf{A}_{R} \mathbf{H}_{p} \mathbf{1}_{N} \text {. }
$$

\section{B. Pilot training with $P C$}

Zadoff-Chu (ZC) pilot sequences are appropriate for LTE systems thanks to their constant amplitude and zero autocorrelation property [12]. A ZC pilot sequence $\boldsymbol{\Phi}$ is defined by its length $N_{Z C}$ and its root number $Q_{Z C}$. Two independent UEs, that simultaneously transmit over the channels $\mathbf{h}_{1}$ and $\mathbf{h}_{2}$ under PC, are assigned the same ZC pilot $\boldsymbol{\Phi}_{\mathrm{PC}}$. With $\mathbf{n}$ being additive white Gaussian noise, the UL signal at the BS is

$$
\mathbf{Y}=\mathbf{h}_{1} \boldsymbol{\Phi}_{\mathrm{PC}}+\mathbf{h}_{2} \boldsymbol{\Phi}_{\mathrm{PC}}+\mathbf{n} .
$$

At the BS, the UL signal is correlated with the complex conjugate of $\boldsymbol{\Phi}_{\mathrm{PC}}$, resulting in

$$
\mathbf{y}_{\mathrm{PC}}=\mathbf{Y} \boldsymbol{\Phi}_{\mathrm{PC}}^{\mathrm{H}}
$$

where ${ }^{\mathrm{H}}$ is the conjugate transpose. In absence of PC, there is a dedicated $\mathbf{y}_{k}$ for each user $k$. This signal is the input of the MPM, described in the next section and shown in Fig. 2.

\section{MPM decontamination and angle estimation}

This section is based on the approach in [6], where the MPM is elaborated on exhaustively. The time-dependent input signal $y(t)$ of the method is of the form

$$
y(t)=x(t)+n(t) \approx \sum_{i=1}^{P} R_{i} e^{s_{i} t}+n(t) \quad \text { for } \quad 0 \leq t \leq T
$$

where $x(t)$ is the desired signal and $n(t)$ is additive white Gaussian noise. The signal can be approximated by a sum 
of $P$ complex exponentials, where $R_{i}$ represents the residues and $s_{i}$ is the complex sum with the damping factors $\alpha_{i}$ as the real part and the angular frequencies $\omega_{i}$ as the imaginary part. After taking $N_{s}$ samples with a sampling period $T_{s}$, the signal can be written as

$$
y\left(l T_{s}\right)=x\left(l T_{s}\right)+n\left(l T_{s}\right) \approx \sum_{i=1}^{P} R_{i} z_{i}^{l}+n\left(l T_{s}\right)
$$

where $l=0,1, \ldots, N_{s}-1$ and with the poles

$$
z_{i}=e^{s_{i} T_{s}}=e^{\left(-\alpha_{i}+j \omega_{i}\right) T_{s}} \quad \text { for } \quad i=1,2, \ldots, P .
$$

With the MPM, the parameters $P, R_{i}$ and $z_{i}$ of this sum of complex exponentials can be estimated. In this paper, we focus on $z_{i}$ because it contains the angular information. Note that estimating $z_{i}$ requires an estimate of $P$ as well, which will be done later in the algorithm. The noisy signal $y(t)$ is allocated in the matrix $\mathbf{Y}_{\text {MPM }}$ as follows

$$
\mathbf{Y}_{\mathrm{MPM}}=\left[\begin{array}{cccc}
y(0) & y(1) & \cdots & y(L) \\
y(1) & y(2) & \cdots & y(L+1) \\
\vdots & \vdots & & \vdots \\
y\left(N_{s}-L-1\right) & y\left(N_{s}-L\right) & \cdots & y\left(N_{s}-1\right)
\end{array}\right] \text {, }
$$

with $L$ the pencil parameter. According to [13], $L$ needs to be chosen between $N_{s} / 3$ and $2 N_{s} / 3$ for efficient noise filtering, with $N_{s}$ being the length of the input signal.

The singular value decomposition of $\mathbf{Y}_{\mathrm{MPM}}$ is carried out as

$$
\mathbf{Y}_{\text {MPM }}=\mathbf{U} \boldsymbol{\Sigma} \mathbf{V}^{\mathrm{H}},
$$

where $\mathbf{U}$ and $\mathbf{V}$ are unitary matrices. The columns of $\mathbf{U}$ contain the left singular vectors and the columns of $\mathbf{V}$ the right singular vectors. $\Sigma$ is a diagonal matrix containing the singular values. At this point in the algorithm, the noise is considered and the parameter $P$ is estimated as $\hat{P}$. To determine the singular values that are valuable for this algorithm, the smallest eigenvalue $\sigma_{\hat{P}}$ needs to be found for which

$$
\frac{\sigma_{\hat{P}}}{\sigma_{\max }}>10^{-p}
$$

where $\sigma_{\max }$ is the largest eigenvalue. In (12), $p$ refers to the amount of significant decimal digits in the input signal. All eigenvalues that are smaller than $\sigma_{\hat{P}}$ are filtered out They are considered noisy and are therefore irrelevant for the reconstruction of the signal. $\mathbf{V}^{\prime}$ is then constructed by keeping only the $\hat{P}$ dominant right-singular vectors of $\mathbf{V}$, i.e.

$$
\mathbf{V}^{\prime}=\left[v_{1}, v_{2}, \ldots, v_{\hat{P}}\right] .
$$

$\mathbf{V}_{1}^{\prime}$ is constructed from $\mathbf{V}^{\prime}$ by removing its last column and $\mathbf{V}_{2}^{\prime}$ by removing its first column. The vector $\hat{\mathbf{z}}$ contains the $\hat{P}$ eigenvalues, which are the poles, and can be found with the 'backward' MPM as

$$
\hat{\mathbf{z}}=\operatorname{eig}\left(\left\{\mathbf{V}_{2}^{\prime}\right\}^{\mathrm{H}}\left\{\left\{\mathbf{V}_{1}^{\prime}\right\}^{\mathrm{H}}\right\}^{+}\right),
$$

where $\{.\}^{+}$indicates the Moore-Penrose pseudo inverse, for a matrix $\mathbf{B}$ defined as

$$
\mathbf{B}^{+}=\left\{\mathbf{B}^{\mathrm{H}} \mathbf{B}\right\}^{-1} \mathbf{B}^{\mathrm{H}} .
$$

According to [14], for a ULA, the angle estimations $\hat{\varphi}$ can be calculated from the imaginary part of the natural logarithm of the poles $\hat{\mathbf{z}}$ as

$$
\hat{\boldsymbol{\varphi}}=\sin ^{-1}\left(\frac{\operatorname{Im}\{\ln (\hat{\mathbf{z}})\}}{(2 \pi / \lambda) d_{H}}\right) .
$$

\section{Implementation of the MPM-based AoA estimation}

The MPM is implemented as described in section II-C. The pencil parameter $L$ is given the value $\left\lfloor N_{s} / 2\right\rfloor$ with $N_{s}$ being the length of the input signal $y_{k}$ for user $k$. Because of the local scattering one-ring model, $N_{s}$ is equal to $M$. In this work we consider a fixed value of $p=1$ in (12), as this gives good results for the broad SNR range considered in this analysis. In theory, the value of $p$ could be determined based on the SNR as signal poles are discarded for high SNRs when using a too low $p$. This is considered a topic for future research. The MPM outputs an array with $\hat{P}$ angle estimations. Because of the threshold set in the MPM, each estimation is considered to be relevant. To determine the best angle estimation $\hat{\varphi}_{k}$ per user, k-means clustering is applied with the number of users $K$, which in these simulations is set to 2 .

\section{E. Channel estimation and NMSE}

For each user $k, \hat{\varphi}_{k}$ is used to estimate the channel $\hat{\mathbf{h}}_{k}$. Again, the local scattering one-ring model from section II-A is applied. The error in the channel estimation is defined by

$$
\tilde{\mathbf{h}}_{k}=\mathbf{h}_{k}-\hat{\mathbf{h}}_{k} \text {. }
$$

Then, the correlation matrix $\mathbf{C}$ of the channel $\mathbf{h}_{k}$ is given by

$$
\mathbf{C}_{k}=\mathrm{E}\left\{\mathbf{h}_{k} \mathbf{h}_{k}^{T}\right\}
$$

where $\mathrm{E}\{$.$\} is the expectation operator. Likewise, the correla-$ tion matrix $\tilde{\mathbf{C}}$ of the error channel is defined as

$$
\tilde{\mathbf{C}}_{k}=\mathrm{E}\left\{\tilde{\mathbf{h}}_{k} \tilde{\mathbf{h}}_{k}^{T}\right\} .
$$

With these expressions, the Normalised Mean Square Error (NMSE) is calculated. We use the NMSE as an indicator of the quality of the channel estimation in this paper, and it is given by

$$
\mathrm{NMSE}_{k}=\frac{\operatorname{tr}\left(\tilde{\mathbf{C}}_{k}\right)}{\operatorname{tr}\left(\mathbf{C}_{k}\right)}
$$

where $\operatorname{tr}($.$) is the trace operator. A perfect estimator results in$ an NMSE of 0 .

\section{Simulation-BASED PERFORMANCE ASSESSMENT}

We carried out a simulations-based evaluation of the performance of the MPM, expressed in terms of the AoA estimation error. In this section this is done for varying $M, \mathrm{SNR}$ and AoA difference. Table I summarises the relevant input parameters used in the simulations. If a parameter differs from this table, it is mentioned. It is important to note that the SNR is always measured at the input of the MPM, to obtain a fair comparison between scenarios with PC and without PC. This is in line with the effective SNR mentioned in [2] where the pilot processing gain is also included. 
TABLE I

INPUT PARAMETERS OF THE SIMULATIONS.

\begin{tabular}{l|r|r|l} 
Parameter & Value & Unit & Meaning \\
\hline$K$ & 2 & - & Number of users \\
$N$ & 8 & - & Number of paths \\
$d_{H}$ & 0.5 & $\lambda$ & Antenna spacing \\
$\sigma$ & 10 & $\circ$ & Standard deviation \\
$Q_{Z C}$ & 3 & - & ZC root number \\
$N_{Z C}$ & 17 & - & ZC sequence length \\
$L=M / 2$ & 32 & - & Pencil parameter
\end{tabular}

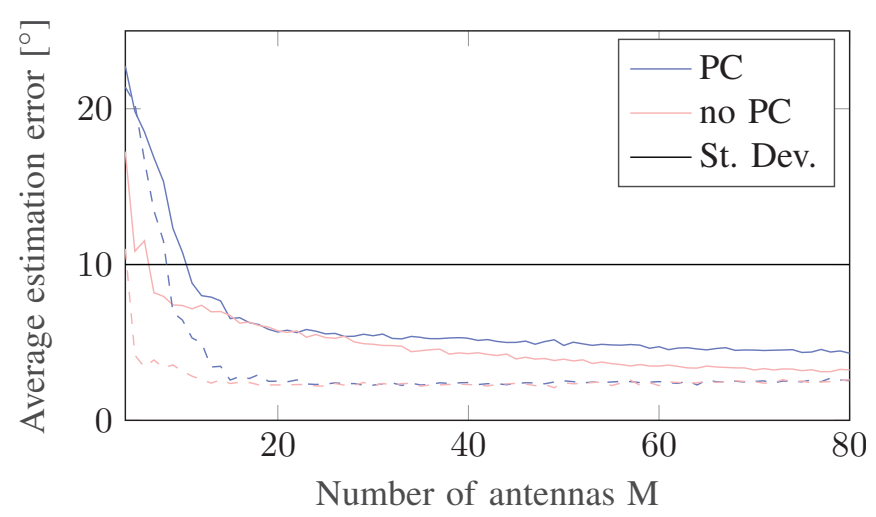

Fig. 3. AoA estimation error for varying $M$ for an SNR of $15 \mathrm{~dB}$ (full line) and $25 \mathrm{~dB}$ (dashed line). The non-PC result is shown as reference as well as the standard deviation $\sigma$ of the angular spread added in the local scattering one-ring model.

\section{A. Performance in terms of number of BS antennas}

First, we analyse the impact of $M$ on the result. The AoA estimation error, averaged over 1000 channel realisations, is plotted in Fig. 3 for both the PC and non-PC scenario with $\varphi_{1}$ and $\varphi_{2}$ respectively $-50^{\circ}$ and $20^{\circ}$. Given the standard deviation of $10^{\circ}$ angular spread and with an SNR of $15 \mathrm{~dB}$ (full lines), a good and stable average estimation error is found starting from $M$ approximately equal to 20 . Hence, our approach is able to, in a PC scenario in massive MIMO, achieve the same accuracy as the reference scenario without PC. For an SNR of $25 \mathrm{~dB}$ (dashed lines), the estimation error is even lower. Considering that practical massive MIMO environments will use more than 20 antennas, the results are certainly favorable.

The AoA estimations are used to estimate the channel, by giving it as an input to the local scattering one-ring model. The quality of the channel is quantified by the NMSE, shown in Fig. 4 for an SNR of $25 \mathrm{~dB}$. For $M=20$, the NMSE is approximately $-50 \mathrm{~dB}$. This means that the estimation error variance is only $0.001 \%$ of the variance of the original channel. Note that the curves in Fig. 3 and 4 are not always decreasing due to the threshold-selected number of valid estimations in (12) and the statistical nature of the data.

\section{B. Performance in terms of SNR}

Fig. 5 shows the estimation error as a function of the SNR with $M=12$ (full line) and $M=64$ (dashed line), averaged over 1000 channel realisations. The results are plotted for two cases: PC and no PC. The angles to estimate, $\varphi_{1}$ and $\varphi_{2}$, are

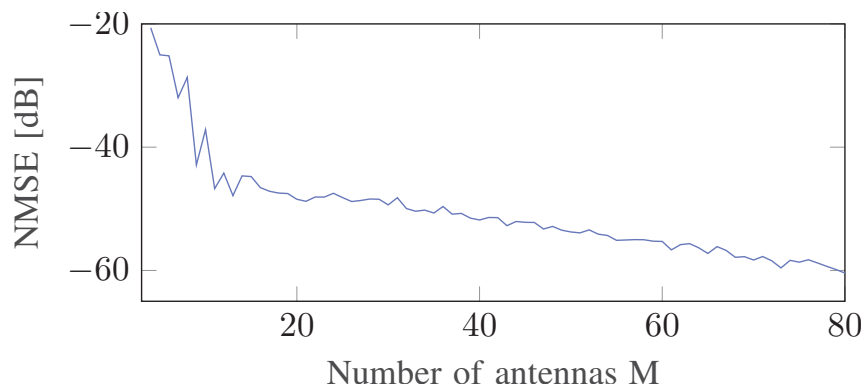

Fig. 4. NMSE results for channel estimation under PC condition based on the estimated angle, as a function of $M$.

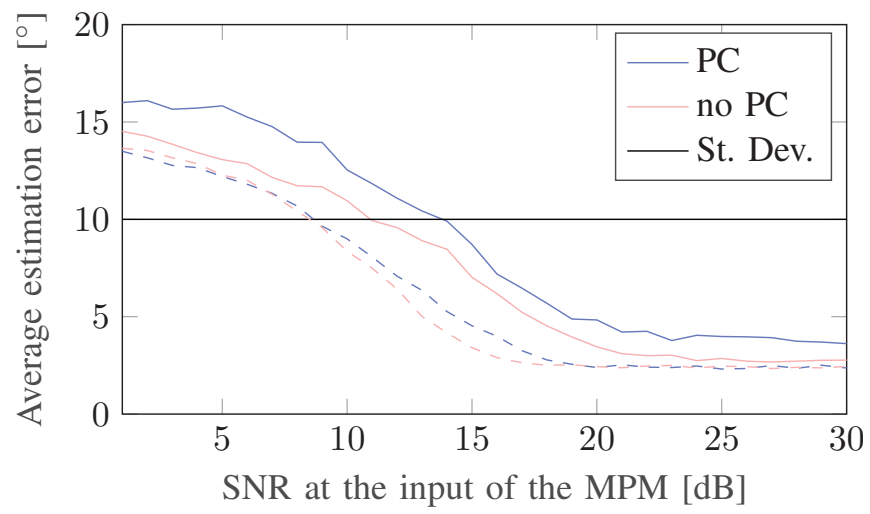

Fig. 5. AoA estimation error as a function of SNR under PC condition due to reused pilots with $M=12$ (dashed) and $M=64$ (full line). The non-PC result is shown as reference as well as the standard deviation $\sigma$ of the angular spread added in the local scattering one-ring model.

$-50^{\circ}$ and $20^{\circ}$, respectively. For a standard deviation of $10^{\circ}$ and an SNR around $20 \mathrm{~dB}$, the estimation error stabilizes around $3^{\circ}$ for no PC and $4^{\circ}$ for PC. Again, the PC scenario results in almost the same accuracy as for the non-PC scenario. While these estimation values may not meet the requirements for very accurate positioning purposes, it is definitely sufficient when a rough estimate of the AoA is needed. The latter is true for the application of this paper, namely the channel estimation based on the estimated AoA; then this accuracy is sufficient. This is verified by calculating the NMSE for this PC result, as reported in section III-A. We achieve an NMSE of -50 dB for SNRs of approximately $15 \mathrm{~dB}$ and higher. We can conclude that the theoretical SNR performance of $20-25 \mathrm{~dB}$ is amply achieved.

\section{Performance for overlapping AoA}

We also considered the challenging case where the two users demonstrate a similar AoA. One user is given a fixed $\varphi_{1}$ of $0^{\circ}$, while $\varphi_{2}$ varies over the range of $\left[-90^{\circ}, 90^{\circ}\right]$. The SNR is $15 \mathrm{~dB}$ and $M$ is 64 . The average estimation error over 1000 channel realisations is plotted for both users as a function of the variable $\varphi_{2}$ in Fig. 6. It shows an obvious peak of estimation errors when the two users have approximately the same AoA of $0^{\circ}$. However, this peak is still beneath the standard deviation of $10^{\circ}$. The distortions on the edges are 


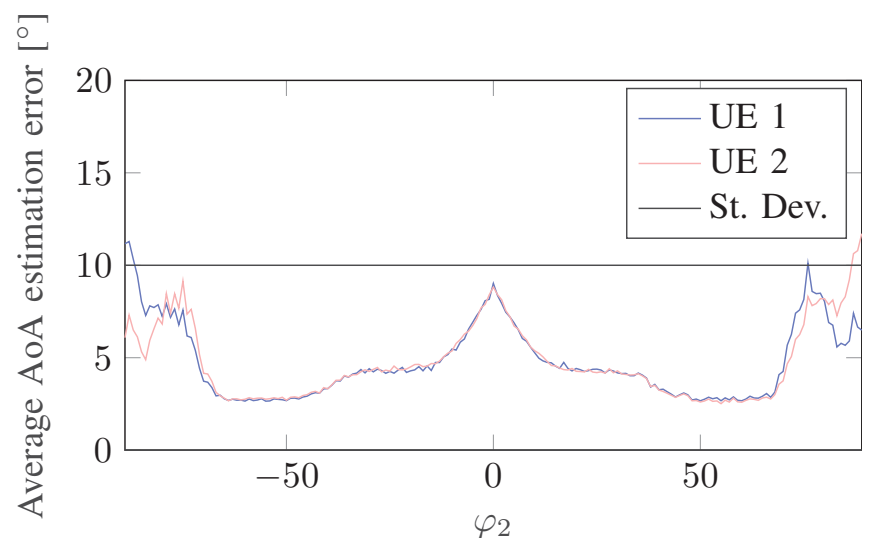

Fig. 6. AoA estimation error for a fixed user on $0^{\circ}$ and variable user under $\mathrm{PC}$ condition for $M=64$ and $\mathrm{SNR}=15 \mathrm{~dB}$.

explained by the added spread angle $\delta_{n}$ in (2), which is chosen uniformly in the interval $[-10 \sqrt{3}, 10 \sqrt{3}]$. The width of the distortions depend on $M$ in the ULA.

\section{EXPERIMENTAL VALIDATION IN A REAL-LIFE TESTBED}

In this experiment, we replace the local scattering one-ring channel model with a real-life indoor channel measurement. The goal is to verify the ability of the MPM to extract the AoA from this captured signal transmitted on an actual channel and thus verifying the assumption of the simplified channel model.

\section{A. Testbed components and data collection}

The available KU Leuven massive MIMO BS from ESAT, described in [15], contains 64 antennas in two rectangular arrays. Table II summarises the most important parameters applied to the testbed during the aforementioned experiment.

TABLE II

PARAMETERS OF THE MASSIVE MIMO TESTBED.

\begin{tabular}{l|r|r|l} 
Parameter & Value & Unit & Meaning \\
\hline$B W$ & 20 & $\mathrm{MHz}$ & Bandwidth \\
$f$ & 2.61 & $\mathrm{GHz}$ & Carrier frequency \\
$S N R$ & $20-25$ & $\mathrm{~dB}$ & Signal-to-Noise Ratio \\
$d_{H}$ & 0.61 & $\lambda$ & Antenna spacing
\end{tabular}

The UE has a directional patch antenna, as described in [16]. The experiments are performed in a room featuring a moderate amount of multipath. The data is collected through the Labview software, where the output channel $\mathbf{H}$ has three dimensions: users, antennas and subcarriers. The subcarrier dimension differs from the simulations, because the Orthogonal Frequency Division Multiplexing (OFDM) modulation scheme was not implemented in the simulation. The scripts for channel collection and channel processing are available online ${ }^{2}$.

\section{B. Antenna selection}

The testbed has 64 antennas in two arrays. However, to be comparable to the simulations, a ULA is needed. Therefore,

\footnotetext{
${ }^{2}$ https://github.com/networkedsystems/MaMIMO_outdoor_expermient-
}

one row of antennas is selected to serve as a ULA. The disadvantage is then that $M$ is limited to 12 , due to the fixed testbed set-up. Experiments show that the two middle rows (called Row2 and Row3) from this arrangement deliver the best results. The outer rows (called Row1 and Row4) suffer from asymmetry because the antennas on the far sides do not experience the same mutual coupling whereas the other antennas do, as shown in [16].

\section{UE placement}

The AoA $\varphi$ of the UE is measured with a protractor and measuring tape. This measurement is a rough estimation of the $\varphi$ with an accuracy of $\pm 2^{\circ}$. Due to the limitation of the room, the range of possible angles is $\left[-10^{\circ}, 30^{\circ}\right]$. In addition, the distance between the UE and the BS is always $1.7 \mathrm{~m}$, which is too low to assume planar wavefronts as the simulations did. Note that the angle estimations need to be interpreted in relation to a reference angle, which was chosen $0^{\circ}$ in this measurement.

\section{AoA estimation for one user}

Numerical results for the measurements described in the previous sections for Row 2 and Row 3 can be found in Table III and IV. The conclusion is that the AoA can indeed be estimated by the MPM, where the estimation error always is in the range of $\left[-4.70^{\circ}, 4.83^{\circ}\right]$, but often it is even smaller. Note that the real AoA is to be considered taking into account the imprecision due to the measurement method.

TABLE III

NUMERICAL RESULTS OF THE MEASUREMENTS FOR ROW2

\begin{tabular}{c|cc|c} 
Real AoA $\left[{ }^{\circ}\right]$ & \multicolumn{2}{|c|}{$\begin{array}{c}\text { AoA estimation }\left[{ }^{\circ}\right] \\
\text { Absolute }\end{array}$} & Est error range $\left[^{\circ}\right]$ \\
\hline-10 & -53.62 & -12.70 & {$[-4.70,-0.70]$} \\
0 & -40.92 & 0 & 0 \\
10 & -27.80 & 13.12 & {$[1.12,5.12]$} \\
20 & -18.09 & 22.83 & {$[0.83,4.83]$} \\
30 & -9.29 & 31.63 & {$[-0.37,3.63]$}
\end{tabular}

TABLE IV

NUMERICAL RESULTS OF THE MEASUREMENTS FOR ROW3.

\begin{tabular}{|c|c|c|c|}
\hline \multirow[t]{2}{*}{ Real AoA $\left[{ }^{\circ}\right]$} & \multicolumn{2}{|c|}{ AoA estimation $\left[{ }^{\circ}\right]$} & \multirow[t]{2}{*}{ Est error range $\left[{ }^{\circ}\right]$} \\
\hline & Absolute & Relative to $0^{\circ}$ & \\
\hline-10 & -25.34 & -12.18 & {$[-4.18,-0.18]$} \\
\hline 0 & -13.16 & 0 & 0 \\
\hline 10 & -1.43 & 11.73 & {$[-0.27,3.73]$} \\
\hline 20 & 7.34 & 20.50 & {$[-1.50,2.50]$} \\
\hline 30 & 16.86 & 30.02 & {$[-1.98,2.02]$} \\
\hline
\end{tabular}

\section{E. AoA estimation for two users}

Adding a virtual second user in this scenario enables investigations for situations with PC. This virtual user is added as follows: the channel of user 1 is the fixed channel of $0^{\circ}$ and the channel of user 2 is variable, using the measured channels for the remaining possible angles. Table $\mathrm{V}$ shows the results of the variable user, when PC occurs with the fixed user. Note that for both users, the reference angle is $0^{\circ}$, causing the estimation 
error for this angle to always be 0. Experiments show the validity of the MPM-based method to estimate the AoA with an accuracy sufficient for channel estimation purposes, as the range is always within $\left[-4.18^{\circ}, 3.73^{\circ}\right]$.

TABLE V

NUMERICAL RESULTS OF THE MEASUREMENTS FOR TWO USERS WITH PC

\begin{tabular}{|c|c|c|c|}
\hline \multirow[t]{2}{*}{ Real AoA $\left[{ }^{\circ}\right]$} & \multicolumn{2}{|c|}{ AoA estimation $\left[{ }^{\circ}\right]$} & \multirow[t]{2}{*}{ Est error $\left[{ }^{\circ}\right]$} \\
\hline & Absolute & Relative to $0^{\circ}$ & \\
\hline-10 & 13.65 & -8.51 & {$\left[\begin{array}{lll}-0.49 & 3.51\end{array}\right]$} \\
\hline 0 & 22.16 & 0 & 0 \\
\hline 10 & 30.49 & 8.33 & {$[-3.67,0.33]$} \\
\hline 20 & 41.55 & 19.39 & {$[-1.39,2.61]$} \\
\hline 30 & 50.16 & 28 & {$[-4.00,0.00]$} \\
\hline
\end{tabular}

\section{CONCLUSION AND FUTURE WORK}

Massive MIMO is a key technology to achieve the demanding peak spectral efficiency requirements of $5 \mathrm{G}$. Leveraging on channel knowledge collected with pilot signals, massive MIMO can send multiple data signals simultaneously to multiple users over the wireless channel. However, due to limited coherence time and bandwidth, the reuse of pilots in the channel estimation phase is inevitable. This reuse results in PC, which generates interference and affects the communication performance.

To mitigate this problem, we propose an MPM-based approach to estimate the AoA from received pilot signals. With the estimated angle, the channel can be estimated more accurately, which is verified by the NMSE. The MPM theoretically offers a good performance for an SNR of 20-25 dB.

We implemented the MPM in a massive MIMO context with PC, evaluating the performance based on a Matlab simulation framework. The channel is modelled by the local scattering one-ring channel model because of its simplicity and direct link to the AoA. The MPM estimates the AoA of the received signal. We interpret the results with k-means clustering. We assessed the performance for varying $M$, SNR and AoA difference, including the non-PC case as a reference. The results show that our method achieves a consistent good performance with 20 antennas or more, while lower values also work well for higher SNRs. This is certainly a favorable result, as practical massive MIMO environments will consider more than 20 antennas. The theoretical performance for SNR values of 20-25 $\mathrm{dB}$ predicted in [7] is outperformed. This is explained by the use of a simplified channel model, directly linked to the AoA. Lastly, higher estimation errors occur when the users have overlapping AoAs, as expected.

Additional real-life indoor measurements were performed to verify the result of the simulations and its assumptions. These measurements are complex due to multiple reasons. Firstly, a ULA of only 12 antennas is available, which is insufficient to acquire optimal results. Secondly, reflections and disadvantageous physical effects play a role now. Lastly, the available angle range is limited to $\left[-10^{\circ} 30^{\circ}\right]$. Due to these complexities, only one user was active during the measurements. A virtual user was added by combining two sequential measurements.
These experiments confirm that the channels suffering PC, can be decorrelated sufficiently.

Overall we conclude that the MPM-based approach presents both a straightforward and a solid solution to estimate the AoA of received signals. In future work a further experimental validation of the promising results under more circumstances would be most interesting.

Apart from performance, other relevant insights were acquired. These include the observation that not all possible ULA's are suitable and that asymmetry of the antennas and mutual coupling play an important role in AoA estimations.

We aim to extend our AoA estimation method to URA, in order to estimate both azimuth and elevation angles, using an updated algorithm. This will broaden the applicability of the method considerably, and allow for more extensive experimental validation in the KU Leuven massive MIMO testbed.

\section{REFERENCES}

[1] E. G. Larsson and L. Van der Perre, Massive MIMO for 5G, IEEE 5G Tech Focus, vol. I, p. 4, 2017.

[2] E. Björnson, J. Hoydis and L. Sanguinetti, Massive MIMO networks. Spectral energy and hardware efficiency, Foundations and Trends in Signal Processing, vol. 11, no. 3-4, pp. 154-655, 2017.

[3] A. Goldsmith, Wireless Communications, Cambridge University Press, 2005.

[4] H. Yin, D. Gesbert, M. Filippou and Y. Liu, A Coordinated Approach to Channel Estimation in Large-Scale Multiple-Antenna Systems, IEEE Journal on Selected Areas in Communications, 31(2), pp. 264-273, 2016.

[5] H. Yin, D. Gesbert, M. C. Filippou and Y. Liu, Decontaminating pilots in massive MIMO systems, IEEE International Conference on Communications (ICC), pp. 3170-3175. 2013.

[6] T. K. Sarkar and O. Pereira, Using the Matrix Pencil Method to estimate the parameters of a sum of complex exponentials, IEEE Antennas and Propagation Magazine, vol. 37, pp. 48-55, February 1995.

[7] Y. Hua, On techniques for estimating parameters of exponentially damped/undamped sinusoids in noise, $\mathrm{PhD}$ thesis, Syracuse University: Electrical Engineering and Computer Science, 1998.

[8] F Hu, T. K. Sarkar and Y. Hua, Utilization of bandpass filtering for the Matrix Pencil Method, IEEE Transactions on Signal Processing, vol. 41, pp. 442, January 1993

[9] K. Chahine, V. Baltazart, Y. Wang, X. Derobert and C. Lebastard, Effects of frequency-dependent attenuation on the performance of time delay estimation techniques using ground penetrating radar, 17th European Signal Processing Conference, pp. 749-753, August 2009.

[10] A. P. Guevara, C. Cheng-Ming and S. Pollin, Pilot contamination in massive MIMO: Virtual angular information aided channel estimation, Presented at the EUCN 2018, Ljubljana, Slovenia, June 2018.

[11] K. I. Pedersen, P. E. Mogensen and B. H. Fleury, Power azimuth spectrum in outdoor environments, Electronics Lett., 33(18), pp. 1583$1584,1997$.

[12] Z. Ali, Z. Chen and F. Yin, Pilot decontamination in TDD multicell massive MIMO systems with infinite number of BS antennas, Canadian Journal of Electrical and Computer Engineering, vol. 40, pp. 171-180, Summer 2017.

[13] Y Hua and T. K. Sarkar, On SVD for estimating generalised eigenvalues of singular matrix pencil in noise, IEEE Transactions on Signal Processing, vol. 39, no. 4, pp. 892-900, April 1991.

[14] K. Aouina Aliouche and D. Benazzouz, Split array of antenna sensors and Matrix Pencil Method for azimuth and elevation angle estimation, IET Signal Processing, vol. 11, no. 6, pp. 687-694, 2017.

[15] A. P. Guevara, C. Chen and S. Pollin, Hardware and Spectrum Sharing for Distributed Massive MIMO 52nd Asilomar Conference on Signals, Systems, pp. 619-623. 2018.

[16] C. Chen, V. Volski, L. Van der Perre, G. A. E. Vandenbosch, and S. Pollin, Finite large antenna arrays for massive MIMO: Characterization and system impact, IEEE Transactions on Antennas and Propagation, vol. 65, pp. 6712- 6720, December 2017. 